\title{
Absorción de N, P, K, Ca y Mg en cultivo de pepino (Cucumis sativus L.) bajo sistema hidropónico
}

\author{
Absorption of N, P, K, Ca and $\mathrm{Mg}$ in cucumber \\ (Cucumis sativus L.) under a hydroponic system
}
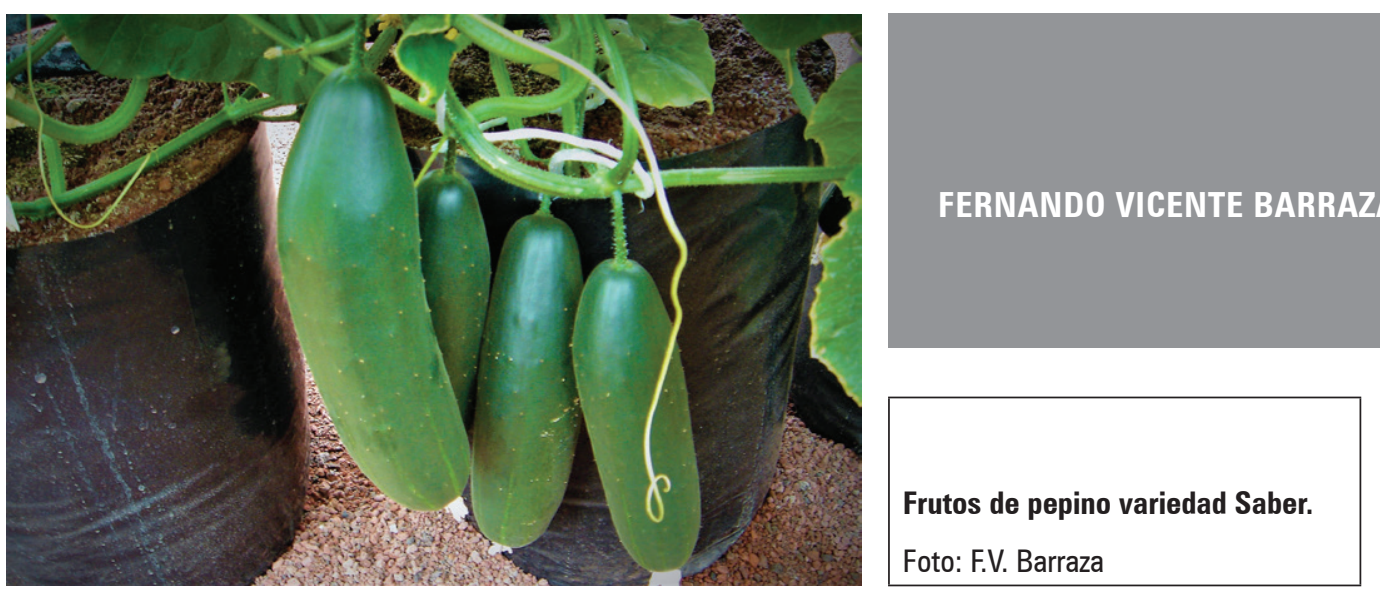

\section{RESUMEN}

En México se cultiva pepino en invernadero con sistema hidropónico de suministro de nutrimentos, mediante diferentes soluciones nutritivas, con concentraciones variables, sin embargo, no se conoce con exactitud la absorción de nutrimentos del cultivo para que el crecimiento y producción sean óptimos. Por lo anterior, se hizo una investigación en la Universidad Autónoma Chapingo (México), con el objetivo de cuantificar la cantidad de macro nutrimentos que absorbe el cultivo. Se utilizó diseño en bloques completos al azar y se evaluaron cuatro concentraciones de la solución nutritiva universal de Steiner: 25, 75, 125 y 175\%. Se cuantificaron los contenidos de N, P, K, Ca y Mg en la materia seca total de plantas y a partir de estos datos se calculó la absorción. Se encontró que la mayor absorción de nutrimentos ocurrió con la solución nutritiva al 175\% de concentración: N 13,055; P 5,730; K 15,143; Ca 37,281 y Mg 9,750 g/planta.

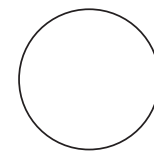

Palabras clave adicionales: absorción mineral de nutrimentos, hortalizas, cucurbitáceas, fertirrigación, invernadero.

\section{ABSTRACT}

In Mexico, cucumber is cultivated in greenhouses with a supply of nutriments using a hydroponic system with different nutrient solutions and variable concentrations. However, the absorption of nutrients for optimum growth and yield is not exactly known. Therefore, this research was conducted at the Universidad Autónoma Chapingo (Mexico) in order to quantify the amount of macro nutriments absorbed by the crops.

\footnotetext{
Facultad de Ciencias Agrícolas, Universidad de Córdoba, Montería (Colombia). ORCID Barraza, F.V.: 0000-0002-36712865

2 Autor para correspondencia: fbarraza@correo.unicordoba.edu.co
} 
Four concentrations of the universal Steiner nutriment solution $(25,75,125$ and $175 \%)$ in a complete randomized block design were used. The contents of $\mathrm{N}, \mathrm{P}, \mathrm{K}, \mathrm{Ca}$ and $\mathrm{Mg}$ were measured in the whole plant dry matter; then, the absorption was calculated. Yield was also evaluated. It was found that the highest nutrient absorption occurred with the nutrient solution at 175\% of concentration: N 13,055; P 5,730; K 15,143; Ca 37,281 and Mg 9,750 g/plant.

Additional key words: mineral nutrient uptake, vegetables, cucurbit, fertirrigation, greenhouse.

Received for publication: 08-02-2017 Accepted for publication: 30-09-2017

INTRODUCCIÓN

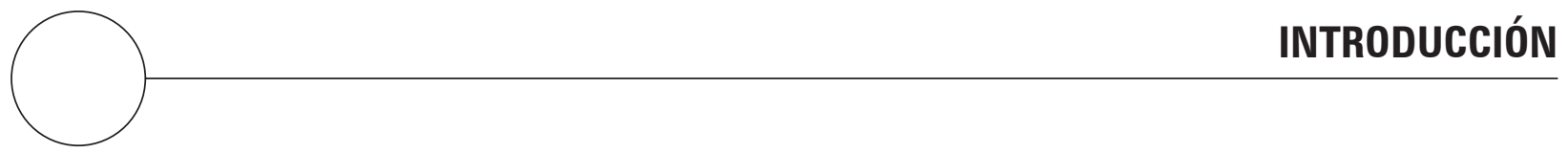

El pepino (Cucumis sativus L.) es una de las hortalizas de mayor importancia económica en el mundo (Bai et al., 2016). Su producción ha aumentado significativamente en los últimos cinco años y en el año 2015 alcanzó el valor más alto en la historia de sus exportaciones, correspondiente a 1.900 millones de Euro (Horto Info, 2016).

Actualmente, México lidera la exportación mundial de pepinos con el $25 \%$ del volumen total exportado y ocupa el primer lugar en el mundo como exportador de esta hortaliza a los Estados Unidos de América, suministrándole el 90\% de su comercialización (Reho, 2015).

Más del $60 \%$ del pepino exportado por México se produce en condiciones de cultivo protegido, en invernaderos con tecnología que incluye el uso de genotipos de alto rendimiento y larga vida de anaquel, siembra de precisión, acolchados, maquinaria especializada para empaque y uso de fertirrigación hidropónica (Reho, 2015).

Como componente tecnológico para aumentar el rendimiento y calidad en cultivos de pepino, la fertirrigación hidropónica utiliza el riego por goteo para aplicar fertilizantes de alta solubilidad y mantener niveles óptimos de nutrimentos en la rizosfera. Teniendo en cuenta que, estos fertilizantes tienden al encarecimiento, provocan impactos negativos en el ambiente y en la salud de los consumidores, cuando son utilizados en cantidades inadecuadas. Actualmente se busca que los sistemas hidropónicos basados en fertirrigación sean más eficientes en cuanto al uso del agua y fertilizantes (De Santiago, 2008; Sánchez et al., 2014).

Uno de los aspectos importantes para aprovechar al máximo los beneficios de la fertirrigación hidropónica sobre el aumento de rendimiento en cultivo de pepino, es el conocimiento de la absorción de nutrimentos minerales que hace la planta para llevar a cabo la acumulación y distribución de materia seca durante su ciclo biológico, la cual está basada principalmente en la habilidad de absorber una cantidad de nutrimentos necesarios para obtener una meta de producción. Esta absorción se cuantifica mediante la concentración de materia seca total o de productos cosechados. Una vez conocidos los requerimientos nutrimentales del cultivo, es posible mantener las cantidades de minerales en óptimas proporciones en la planta y aplicarlos a través de una solución nutritiva, con base en una planificación adecuada de la fertilización (Anjanappa et al., 2012; Carmona et al., 2015).

De acuerdo con Sívori (1986) el análisis de plantas muestra la presencia de más de 90 elementos minerales, de los cuales, cualquiera que se encuentre en el medio en que crezcan las raíces, puede formar parte de la composición de los vegetales. Sin embargo, no todos son necesarios para el crecimiento y desarrollo normales de las plantas y se ha encontrado que solamente 18 son esenciales. Los elementos esenciales se pueden clasificar como macro nutrimentos y micro nutrimentos. Los macro nutrimentos son usados por las plantas en grandes cantidades y pueden ser estructurales como el $\mathrm{C}$ (obtenido del $\mathrm{CO}_{2}$ del aire), $\mathrm{H}$ y O (obtenidos del agua), primarios como el N, P, y K y secundarios como Ca, Mg y S. Los micro nutrimentos son utilizados en pequeñas cantidades y son: $\mathrm{Fe}, \mathrm{Cu}$, Cl, Mn, Mo, Zn, Co y Ni (Cornell University, 2010).

Teniendo en cuenta que, en los últimos años han ocurrido cambios importantes en las prácticas de producción del cultivo de pepino; como por ejemplo, la introducción de nuevos genotipos, uso de coberturas 
de polietileno, riego por goteo, espaciamiento entre plantas y diversas recomendaciones en el manejo de la fertilización (Hochmuth y Hanlon, 2013; Feleafel et al., 2014), se hace necesario llevar a cabo estudios en los cuales se cuantifique la absorción de nutrimentos que el cultivo exige, para expresar de manera óptima su potencial productivo, sobre todo en sistemas de agricultura protegida, con el fin de evitar aplicaciones deficientes o excesivas de nutrimentos.

Por lo anterior, el objetivo de la presente investigación fue analizar el efecto de cuatro concentraciones de la solución nutritiva universal de Steiner: 25, 75, 125 y $175 \%$ sobre la absorción de N, P, K, Ca y Mg de un cultivo de pepino bajo condiciones de invernadero con sistema de riego hidropónico.

\section{MATERIALES Y MÉTODOS}

Este trabajo se realizó en condiciones de invernadero tipo diente de sierra, cubierta transparente de polietileno y ventilación cenital, lateral y frontal en la Universidad Autónoma Chapingo, México, 19²9'23" N y 98 $53^{\prime} 37^{\prime \prime} W$, altitud $2.250 \mathrm{msnm}$, durante los meses de febrero y junio de 2015.

El diseño experimental fue en bloques completos al azar con cuatro concentraciones de la solución nutritiva universal de Steiner: 25, 75, 125 y 175\% (tratamientos) y tres repeticiones. Las soluciones nutritivas se aplicaron mediante riego por goteo cuyos componentes fueron: tanque plástico de $200 \mathrm{~L}$ de capacidad para cada solución, tubería porta regantes en policluro de vinilo (PVC) de $25,4 \mathrm{~mm}$ de diámetro acoplada a cada tanque, llave de paso en PVC acoplada a cada tanque y cintas de goteo en polietileno con gasto por emisor de $1 \mathrm{~L} \mathrm{~h}^{-1}$ acopladas a la tubería porta regantes de cada tanque.

La aplicación de las soluciones nutritivas, instalación del experimento y manejo del cultivo se hizo con base en la metodología de Barraza (2012), con producción de plántulas mediante sistema de siembra de semillas de la variedad Saber en charolas germinadoras (Moreno et al., 2011) utilizando sustrato de turba vegetal (Comercializadora Hydro Environment, Tlalnepantla, México), con trasplante a los 25 días después de la siembra (dds) a bolsas negras de polietileno con capacidad de $0,06 \mathrm{~m}^{3}$ de sustrato tezontle rojo y colocando las bolsas en arreglo topológico de 1,5 m entre hileras y 0,30 m entre plantas, para un total de 156 plantas que ocuparon una superficie de $99 \mathrm{~m}^{2}$, sin realización de podas y con tutorado de espaldera de malla plástica de cuadrícula de $10 \mathrm{~cm}$.

Para aplicar el enfoque funcional del análisis de crecimiento vegetal (Gardner et al., 1990; Barraza, 2012), se realizaron muestreos cada $15 \mathrm{~d}$, a tres plantas tomadas al azar, una en cada repetición y se tomaron datos de las variables materia seca total y contenido de macro nutrimentos ( $\mathrm{N}, \mathrm{P}, \mathrm{K}, \mathrm{Ca}$ y $\mathrm{Mg}$ ) en la materia seca total. Las plantas se extrajeron de las bolsas, sin raíz y se secaron en estufa Blue-M ${ }^{\circledR}$ POM-326-F (Thermal Product Solutions, New Columbia, PA, USA), a $65^{\circ} \mathrm{C}$ hasta obtener peso constante. La materia seca total en gramos se obtuvo del promedio de las tres plantas indicadas, pesadas en balanza digital Sartorius 1205 MP ${ }^{\circledR}$ (Sartorius AG, Göttingen, Alemania).

La determinación del contenido de macro nutrimentos se hizo en el laboratorio de nutrición vegetal del Colegio de Posgraduados de Montecillo, México, a partir de muestras de $100 \mathrm{~g}$ de materia seca total, molidas con equipo Wiley Mill Thomas ${ }^{\circledR}$ ED-5 (Arthur H. Thomas Co, Philadelphia, PA, USA). En concordancia con Morillo et al. (2009) el contenido de N se determinó con destilador micro Kjeldahl DEK-1 (Arthur H. Thomas, Philadelphia, PA, USA) y se reportó en porcentaje. Los contenidos de $\mathrm{P}, \mathrm{K}, \mathrm{Ca}$ y $\mathrm{Mg}$ se determinaron por espectrometría de inducción de plasma acoplado, con equipo Varian (Walnut Creek, CA, USA) y se reportaron en $\mathrm{mg} \mathrm{kg}^{-1}$.

La absorción de macro nutrimentos se calculó a partir de los datos obtenidos de contenidos en materia seca, de acuerdo con lo reportado por Anjanappa et al. (2012), mediante las ecuaciones 1 y 2 , y expresado en $\mathrm{g} /$ planta:

$$
\text { Absorción } N(g)=\frac{(\text { materia seca total }) *(\% N)}{100}
$$

$$
\begin{gathered}
\text { Absorción } P, K, \\
C a, M g(m g)
\end{gathered}=\frac{\begin{array}{c}
(\text { materia seca total }) *(m g / k g \\
\text { del nutrimento })
\end{array}}{1000}
$$

A las variables estudiadas se les hizo análisis de varianza y pruebas de comparación de medias de Tukey $(P \leq 0,05)$ y se ajustaron mediante el procedimiento de regresión no lineal PROC NLIN con el software SAS 9.0 (SAS Institute, Cary, NC), seleccionando el modelo logístico, cuya ecuación 3 es la siguiente: 


$$
y=\frac{A}{\left(1+B \cdot e^{-C x}\right)}
$$

donde

y: absorción del nutrimento;

A: máximo valor observado en la absorción del nutrimento;

$B$ : no tiene significado biológico, y solo toma lugar en el tiempo inicial, cuando $x=0$;

$X: \quad$ días después del trasplante (ddt);

$C$ : parámetro relacionado con el valor de $x$ para el punto de inflexión;

$e$ : constante matemática, base del logaritmo natu$\mathrm{ral}=2.718281828$.

\section{RESULTADOS Y DISCUSIÓN}

\section{Absorción de macronutrimentos}

La cuantificación de los macro nutrimentos absorbidos tuvo ajuste al modelo logístico (Tab. 1) y según lo indicado por Alonso et al. (2003), Opara et al. (2012), Nikolic et al. (2012) y Hochmuth et al. (2015) su trayectoria gráfica corresponde al tipo sigmoide. La utilidad práctica de ello consiste en que, a partir de los modelos matemáticos obtenidos se puede estimar de manera precisa y confiable la cantidad de macronutrimentos que se absorben durante el ciclo del cultivo. Este comportamiento tiene fundamento en las bases

Tabla 1. Modelos matemáticos para estimar absorción de macro nutrimentos en cultivo de pepino 'Saber', en cuatro concentraciones de la solución nutritiva universal de Steiner.

\begin{tabular}{|c|c|c|}
\hline $\begin{array}{l}\text { Concentración de la } \\
\text { solución nutritiva (\%) }\end{array}$ & Modelo matemático & $R^{2}$ \\
\hline \multicolumn{3}{|c|}{$\mathrm{N}$} \\
\hline 25 & $y=5,03305 /\left(1+286,277 \mathrm{e}^{-0,071527^{*} \mathrm{ddt}}\right)$ & 0,97 \\
\hline 75 & $y=11,9064 /\left(1+84,4068 \mathrm{e}^{-0,049350^{*} \mathrm{dt}}\right)$ & 0,99 \\
\hline 125 & $y=15,7085 /\left(1+65,1063 \mathrm{e}^{-0,041716^{*} d t}\right)$ & 0,98 \\
\hline 175 & $y=11,4290 /\left(1+152,715 \mathrm{e}^{-0,061842^{*} d \mathrm{dt}}\right)$ & 0,96 \\
\hline \multicolumn{3}{|c|}{$P$} \\
\hline 25 & $y=0,37914 /\left(1+153,545 \mathrm{e}^{-0,076837^{*} d \mathrm{dt}}\right)$ & 0,96 \\
\hline 75 & $y=2,58637 /\left(1+83,1829 \mathrm{e}^{-0,044845^{*} d t}\right)$ & 0,98 \\
\hline 125 & $y=2,77196 /\left(1+33,7194 \mathrm{e}^{-0,038520^{*} d t}\right)$ & 0,98 \\
\hline 175 & $y=6,66015 /\left(1+120,149 \mathrm{e}^{-0.048428^{*} d t}\right)$ & 0,98 \\
\hline \multicolumn{3}{|c|}{$\mathrm{K}$} \\
\hline 25 & $y=5,51979 /\left(1+47,5920 \mathrm{e}^{-0,042575^{*} d t}\right)$ & 0,98 \\
\hline 75 & $y=11,8651 /\left(1+97,2039 \mathrm{e}^{\left.-0,054326^{*} \mathrm{ddt}\right)}\right.$ & 0,99 \\
\hline 125 & $y=21,8935 /\left(1+84,5903 \mathrm{e}^{-0,039564^{*} d t}\right)$ & 0,98 \\
\hline 175 & $y=17,5572 /\left(1+69,9517 \mathrm{e}^{\left.-0.045578^{*} \mathrm{ddt}\right)}\right.$ & 0,99 \\
\hline \multicolumn{3}{|c|}{$\mathrm{Ca}$} \\
\hline 25 & $y=7,00075 /\left(1+300,439 \mathrm{e}^{\left.-0,071125^{*} \mathrm{ddt}\right)}\right.$ & 0,98 \\
\hline 75 & $y=23,9717 /\left(1+409,73 \mathrm{e}^{-0,067012^{*} \mathrm{ddt}}\right)$ & 0,98 \\
\hline 125 & $y=33,6170 /\left(1+178,509 \mathrm{e}^{\left.-0.058300^{*} \mathrm{ddt}\right)}\right.$ & 0,98 \\
\hline 175 & $y=39,7890 /\left(1+188,566 \mathrm{e}^{-0,056609^{*} d \mathrm{dt}}\right)$ & 0,99 \\
\hline \multicolumn{3}{|c|}{$\mathrm{Mg}$} \\
\hline 25 & $y=3,43315 /\left(1+231,581 e^{-0,066991^{*} d d t}\right)$ & 0,96 \\
\hline 75 & $y=6,31009 /\left(1+148,042 e^{-0,0580577^{*} d t}\right)$ & 0,98 \\
\hline 125 & $y=8,52651 /\left(1+135,004 \mathrm{e}^{\left.-0,052573^{*} \mathrm{ddt}\right)}\right.$ & 0,99 \\
\hline 175 & $y=8,38580 /\left(1+222,933 \mathrm{e}^{-0,060783^{*} d t}\right)$ & 0,97 \\
\hline
\end{tabular}

y: absorción (g/planta); ddt: días después del trasplante; $R^{2}$ : coeficiente de determinación. 
fisiológicas del proceso de crecimiento vegetal, ya que la absorción de macro nutrimentos sólo ocurre cuando hay crecimiento de las plantas, debido a que los elementos minerales son esenciales para formar nuevas moléculas y células (Anjanappa et al., 2012).

Además de lo anterior se estableció que, a mayor concentración de las soluciones nutritivas, se presentó mayor absorción de macro nutrimentos (Tab. 2). De tal manera que con la concentración de 175\% hubo mayor absorción de N, P, K, Ca y Mg, superando con diferencias estadísticas significativas a las concentraciones de 25, 75 y $125 \%$ (Tab. 2).

Respecto a la absorción de macro nutrimentos mayores en relación con las concentraciones de la solución nutritiva utilizadas, se encontró que la absorción de $\mathrm{N}$ y K fue mayor que la absorción de P lo que está en concordancia con lo indicado por Gómez et al. (2003), quienes señalan que en la medida que se maximiza el crecimiento del cultivo de pepino, se presenta una mayor demanda de dichos macro nutrimentos, principalmente por la actividad fotosintética. Al respecto, Anjanappa et al. (2012) han encontrado diferencias significativas en la producción de materia seca total del cultivo cuando ha ocurrido aumento en la $a b-$ sorción de $\mathrm{N}$ y K y en concordancia con estudios de Barraza (2012), con la aplicación de solución nutritiva de Steiner al 75, 125 y 175\% de concentración, la acumulación de materia seca total superó en $55 \%$ a la obtenida cuando se aplicó la solución nutritiva al 25\% de concentración.

Con relación a $\mathrm{P}$ se observó que, con el suministro de solución nutritiva al $25 \%$ de concentración, hubo absorción significativamente menor al compararla con la absorción ocurrida cuando se aplicaron las concentraciones de 75, 125 y 175\%. Esto ocurre en el cultivo de pepino debido a que el insuficiente o nulo suministro de $\mathrm{P}$ trae como consecuencia poco crecimiento y atrofia del sistema de raíces, con lo que también ocurre disminución en la absorción de otros nutrimentos, especialmente Ca (Zhang y Shi, 2008). En concordancia con lo anterior, se pudo establecer que la absorción de Ca se vio disminuida significativamente con la solución nutritiva al 75\% de concentración (Tab. 2), lo cual indica que en dicha condición nutrimental también hubo suministro deficiente del macro nutrimento.

Al comparar la absorción de Ca con la absorción de $\mathrm{N}, \mathrm{P}, \mathrm{K}$ y Mg se encontró que, con todas las concentraciones de la solución nutritiva universal de Steiner aplicadas, la mayor absorción nutrimental del cultivo correspondió a Ca (Tab. 2), lo cual está en concordancia con lo indicado por Adams y Hand (2015), en el sentido de que las plantas de pepino absorben considerable contenido de dicho nutrimento en la medida que sus distintos órganos presenten mayor tasa de crecimiento. Para el caso de los frutos, la acumulación de Ca durante su proceso de crecimiento y desarro1lo, contribuye a mayor expansión y acumulación de agua, además de, su contribución en mitigar la pérdida de firmeza que puede ocurrir por las labores de cosecha, manipulación y pos cosecha (Engelkes et al., 1990).

En lo que respecta a $\mathrm{Mg}$, se observaron síntomas de deficiencia en las hojas más viejas de las plantas, cultivadas con la solución nutritiva universal de Steiner al $25 \%$ de concentración. Se presentó pérdida del color verde, clorosis intervenal y secamiento de la lámina foliar que inició desde el borde de la hoja (Fig. 1). En relación con lo indicado por Carmona et al. (2015) la sintomatología descrita está relacionada con daños severos de los tilacoides y rompimiento de los cloroplastos, lo que afecta la fotosíntesis, acumulación de materia seca y rendimiento del cultivo.

Tabla 2. Comparación de medias de la absorción de macro nutrimentos ( $\mathrm{g} / \mathrm{planta}$ ) del pepino 'Saber', en cuatro concentraciones de la solución nutritiva universal de Steiner.

\begin{tabular}{|l|c|c|c|c|c|}
\hline \multirow{2}{*}{ Concentración de la solución } & \multicolumn{5}{|c|}{ Absorción (g/planta) } \\
\cline { 2 - 7 } & $\mathrm{N}$ & $\mathrm{P}$ & $\mathrm{K}$ & $\mathrm{Ca}$ & $\mathrm{Mg}$ \\
\hline $25 \%$ & $5,471 \mathrm{c}$ & $0,489 \mathrm{c}$ & $4,889 \mathrm{c}$ & $6,227 \mathrm{~d}$ & $4,174 \mathrm{c}^{\dagger}$ \\
\hline $75 \%$ & $10,836 \mathrm{~b}$ & $2,173 \mathrm{~b}$ & $11,697 \mathrm{~b}$ & $24,851 \mathrm{c}$ & $6,412 \mathrm{~b}$ \\
\hline $125 \%$ & $11,588 \mathrm{~b}$ & $2,634 \mathrm{~b}$ & $12,446 \mathrm{~b}$ & $33,404 \mathrm{~b}$ & $7,806 \mathrm{~b}$ \\
\hline $175 \%$ & $13,055 \mathrm{a}$ & $5,730 \mathrm{a}$ & $15,143 \mathrm{a}$ & $37,281 \mathrm{a}$ & $9,750 \mathrm{a}$ \\
\hline
\end{tabular}

Promedios con letras distintas en la misma columna indican diferencias significativas según la prueba de Tukey $(P \leq 0,05)$. 
De acuerdo con los resultados obtenidos, se puede inferir que la mayor absorción de macro nutrimentos observada con la aplicación de solución nutritiva universal de Steiner al 175\% de concentración, tiene influencia en el crecimiento del cultivo en razón a los planteamientos de Rauthan y Schnitzer (1981) y Parra et al. (2009), ya que se maximizan variables como altura de planta, longitud de raíces, número de ramificaciones, hojas y flores por planta y acumulación de materia seca total, y de acuerdo con Barraza (2012) con la aplicación de las solución nutritiva universal de Steiner al 25, 75 y 125\% de concentración, el cultivo de pepino disminuyó el rendimiento en 60, 22 y $15 \%$ con respecto al rendimiento obtenido con la solución nutritiva al $175 \%$, el cual fue de $8,20 \mathrm{~kg}$ por planta. Esto muestra que a medida que se disminuyó el suministro de macro nutrimentos, también lo hizo la absorción. Por lo que no pudo ocurrir la expresión adecuada del potencial productivo, que es posible obtener cuando se hace mayor suministro de macro nutrimentos. Estos suministros se absorbieron en mayor cantidad en el cultivo con la condición nutrimental propiciada con la solución al 175\%. Y además de lo anterior, de acuerdo con Halitligil et al. (2002) y Van Eerd y O'Reilly (2009) se ha encontrado que, junto con la disminución del rendimiento, se obtienen frutos de baja calidad.

Para señalar la importancia del efecto que tuvo el aumento del suministro de nutrimentos al cultivo de pepino a través, del aumento en la concentración de la solución nutritiva universal de Steiner (con lo cual se observó respuesta diferencial de aumento en la absorción), es importante resaltar el papel que juegan

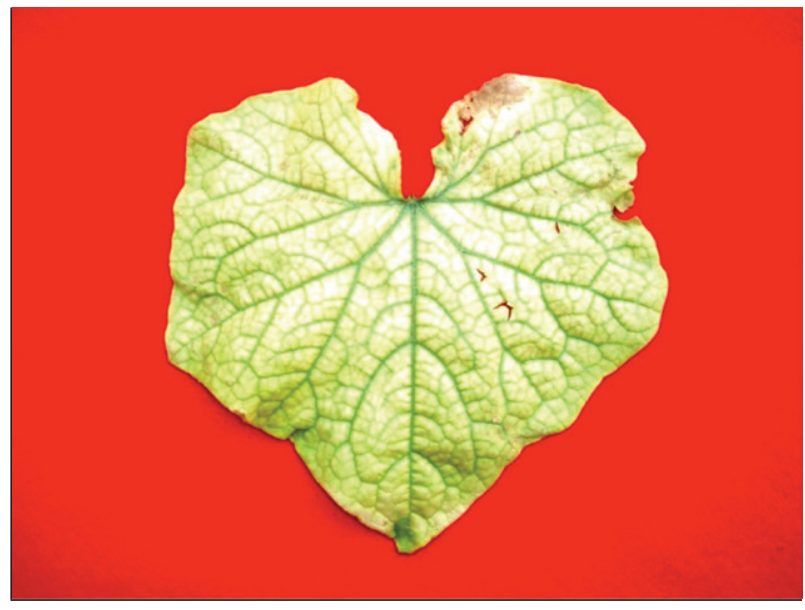

Figura 1. Síntomas de deficiencia de Mg en hoja de pepino cultivado con solución nutritiva universal de Steiner al $25 \%$ de concentración. Foto: F.V. Barraza. los macronutrimentos en la fisiología del proceso de crecimiento y desarrollo del cultivo. En este sentido, $\mathrm{N}$ es el principal nutrimento limitante de dichos procesos, debido a que es el mayor constituyente de proteínas, nucleótidos, clorofila y numerosos metabolitos y componentes celulares (Borlotti et al., 2012; Hochmuth y Hanlon, 2013). De acuerdo con Farag et al. (2010) su influencia en el aumento del rendimiento se debe principalmente a la estimulación de la actividad meristemática y acumulación de metabolitos sintetizados, lo cual produce mayor acumulación de materia seca. Cuando $\mathrm{N}$ es deficiente en el cultivo ocasiona crecimiento achaparrado de las plantas, hojas cloróticas debido a la poca fotosíntesis, floración prematura y acortamiento del ciclo biológico (Danesh et al., 2012).

Con respecto a P, Feleafel et al. (2014) indican que tiene influencia significativa en el establecimiento vigoroso del cultivo de pepino y, contribuye a superar el gasto energético que ocurre cuando se efectúa el trasplante, acelerando el crecimiento y fortalecimiento del sistema radicular. Su importancia sobre el aumento del rendimiento del cultivo, radica en el efecto que tiene sobre el aumento en la producción de frutos (Ortas, 2010; Ghehsareh et al., 2011).

Para el caso de K, Jilani et al. (2009) y Prajapati y Modi (2016) indican que influye favorablemente en el crecimiento, principalmente por el aumento que provoca en la longitud de las raíces, tallo principal, número de ramas, flores y frutos. Además, interviene en el incremento del tamaño y calidad de los frutos y promueve el desarrollo de las semillas, ya que la planta requiere $\mathrm{K}$ en sus procesos metabólicos, como precursor para la formación de celulosa y clorofila y para facilitar la acción de las enzimas que ayudan a la síntesis y translocación de carbohidratos, promoviendo el aumento del rendimiento del cultivo.

En cuanto a Ca, en concordancia con lo indicado por Kazemi (2013), incrementa significativamente el crecimiento reproductivo y rendimiento, manteniendo la calidad y firmeza de los frutos, ya que esta condición es fundamental para mantener la integridad de las membranas celulares y la regulación del transporte de iones (Dabuxilatu, 2005).

En lo concerniente a Mg, Ghehsareh et al. (2011) y Zhu et al. (2015) indican que es el elemento básico que constituye la clorofila e influye directamente en el adecuado funcionamiento de los cloroplastos, granas y lamelas para realizar la fotosíntesis. También, se 
requiere para maximizar el rendimiento y calidad de frutos, a los que confiere rigidez y color verde oscuro (Valenzuela et al., 1994).

\section{CONCLUSIONES}

La mayor respuesta significativa en la absorción de macro nutrimentos se observó con la aplicación de la solución nutritiva universal de Steiner al $175 \%$ de concentración. En comparación con las concentraciones de la solución al 25, 75 y $125 \%$, se recomienda solo la utilización de la solución nutritiva al 175\% de concentración para obtener la expresión más adecuada del potencial productivo del cultivo de pepino sembrado en invernadero con sistema hidropónico de suministro de macro nutrimentos.

Conflicto de intereses: el manuscrito fue preparado y revisado por el autor, quien declara no tener algún conflicto de interés que coloquen en riesgo la validez de los resultados aquí presentados.

\section{REFERENCIAS BIBLIOGRÁFICAS}

Adams, P. y D. Hand. 2015. Effects of humidity and Ca level on dry-matter and $\mathrm{Ca}$ accumulation by leaves of cucumber (Cucumis sativus L.). J. Hort. Sci. 68, 767774. Doi: 10.1080/00221589.1993.11516411

Alonso, M., L. Tijerina, P. Sánchez, A. Martínez, L. Aceves y J. Escalante. 2003. Modelo logístico: herramienta para diagnosticar el cuánto y cuándo fertirrigar. Terra Lat. 21, 225-231.

Anjanappa, M., J. Venkatesha y B. Kumara. 2012. Dry matter accumulation and uptake of nutrients by cucumber (cv. Hassan Local) as influenced by organic, inorganic and bio- fertilizers. Karnataka J. Agric Sci. 25(4), 552-554.

Bai, L., H. Deng, X. Zhang, X, Yu e Y. Li. 2016. Gibberellin is involved in inhibition of cucumber growth and nitrogen uptake at suboptimal root-zone temperatures. PLoS ONE 11(5), e0156188. Doi: 10.1371/journal. pone.0156188

Barraza, F.V. 2012. Acumulación de materia seca del cultivo de pepino (Cucumis sativus L.) en invernadero. Temas Agrarios 17(2), 18-29.

Borlotti, A., G. Vigani y G. Zocchi. 2012. Iron deficiency affects nitrogen metabolism in cucumber (Cucumis sativus L.) plants. BMC Plant Biol. 12(189), 1-15. Doi: 10.1186/1471-2229-12-189

Carmona, V., L. Costa y A. Filho. 2015. Symptoms of nutrient deficiencies on cucumbers. Int. J. Plant Soil Sci. 8(6), 1-11. Doi: 10.9734/IJPSS/2015/20243
Cornell University. 2010. Basic concepts of plant nutrition. En: NRCCA, https://nrcca.cals.cornell.edu/nutrient/ CA1/CA010102.php; consulta: septiembre de 2016.

Dabuxilatu, M. 2005. Interactive effect of salinity and supplemental calcium application on growth and ionic concentration of soybean and cucumber plants. J. Soil Sci. Plant Nutr. 51(4), 549-555. Doi: 10.1111/j.17470765.2005.tb00063.x

Danesh, R., S. Bidarigh, E. Azarpour, M. Moraditochaee y H. Bozorgi. 2012. Study effects of nitrogen fertilizer management and foliar spraying of marine plant Ascophyllum nodosum extract on yield of cucumber ( $\mathrm{Cucu}$ mis sativus L.). Intl. J. Agri. Crop Sci. 4(20), 1492-1495.

De-Santiago, J. 2008. Sistemas productivos mediante el manejo de fertirrigación. En: Meister Media Worldwide, http://www.hortalizas.com/miscelaneos/sistemas -productivos-mediante-el-manejo-de-fertirrigacion; consulta: agosto de 2016.

Engelkes, C., I. Widders y H. Price. 1990. Ontogenetic changes in and content in pickling influenced by genotype calcium concentration cucumber fruit as and environment. J. Amer. Soc. Hort. Sci. 115(4), 555-558.

Farag, A., M. Abdrabbo y M. Hassanein. 2010. Response of cucumber for mulch colors and phosphorus levels under greenhouse. Egypt. J. Hort. 37, 53-64.

Feleafel, M., Z. Mirdad y A. Hassan. 2014. Effecte of NPK fertigation rate and starter fertilizer on the growth and yield of cucumber grown in greenhouse. J. Agric. Sci. 6(9), 81-92. Doi: 10.5539/jas.v6n9p81

Gardner, F., R. Pearce y R. Mitchell. 1990. Physiology of crop plants. $2^{\text {nd }}$ ed. Iowa University Press, Ames, IA, USA.

Ghehsareh, A., S. Khosravan y A. Shahabi. 2011. The effect of different nutrient solutions on some growth indices of greenhouse cucumber in soilless culture. J. Plant Breed. Crop Sci. 3(12), 321-326.

Gómez, M., M. Baille, M. González y J. Mercader. 2003. Comparative analysis of water and nutrient uptake of glasshouse cucumber grown in NFT and perlite. Acta Hortic. 614, 175-180. Doi: 10.17660/ ActaHortic.2003.614.24

Halitligil, M., A. Akin, H. Kislal, A. Ozturk y A. Deviren. 2002. Yield, nitrogen uptake and nitrogen use efficiency by tomato, pepper, cucumber, melon and eggplant as affected by nitrogen rates applied with drip-irrigation under greenhouse conditions. Int. Atomic Agency Tech. Doc. 1266, 99-110.

Hochmuth, G. y E. Hanlon. 2013. A summary of N, P, and $K$ research with cucumber in Florida. En: Institute of Food and Agricultural Sciences (IFAS Extension), University of Florida, http://edis.ifas.ufl.edu/cv226; consulta: septiembre de 2016 .

Hochmuth, G., R. Mylavarapu y E. Hanlon. 2015. The four Rs of fertilizer management. pp. 5-7. En: Morgan, K. 
(ed.). Nutrient management of vegetable and row crops handbook. Institute of Food and Agricultural Sciences (IFAS), University of Florida, Gainesville, FL, USA.

Horto Info. 2016. La producción mundial de pepino supera los 65 millones de toneladas. En: http://www.hortoinfo.es/index.php/4693-prod-mund-pepino-020614; consulta: agosto de 2016 .

Hyams, D. 2003. Curve expert 1.3. A comprehensive curve fitting system for Windows ${ }^{\odot}$. Unregistered evaluation copy. Starkville, MS, USA.

Jilani, M., A. Bakar, K. Waseem y M. Kiran. 2009. Effect of different levels of NPK on the growth and yield of cucumber (Cucumis sativus) under the plastic tunnel. J. Agric. Soc. Sci. 5(3), 99-101.

Kazemi, M. 2013. Response of cucumber plants to foliar application of calcium chloride and paclobutrazol under greenhouse conditions. Bull. Env. Pharmacol. Life Sci. 2(11), 15-18.

Moreno, E., F. Sánchez, L. González, C. Pérez y N. Magaña. 2011. Efectos del volumen de sustrato y niveles de N-P-K en el crecimiento de plántulas de pepino. Terra Lat. 29(1), 57-63.

Morillo, G., R. Monsalve, J. Mendoza, D. Isea, I. Araujo, L. Vargas y N. Angulo. 2009. Evaluación química y microbiológica del pepino (Cucumis sativus L.) cultivado con aguas residuales. Rev. Téc. Ing. Univ. Zulia 32(1), 68-76.

Nikolic, M., S. Cesco, R. Monte, N. Tomasi, S. Gottardi, A. Zamboni, R. Pinton y Z. Varanini. 2012. Nitrate transport in cucumber leaves is an inducible process involving an increase in plasma membrane $\mathrm{H}^{+}$-ATPase activity and abundance. BMC Plant Biol. 12(66), 1-12. Doi: 10.1186/1471-2229-12-66

Opara, E., K. Zuofa, N. Isirimah y D. Douglas. 2012. Effects of poultry manure supplemented by NPK 15:15:15 fertilizer on cucumber (Cucumis sativus L.) production in Port Harcourt (Nigeria). Afr. J. Biotechnol. 11, 10548-10554. Doi: 10.5897/AJB11.1356

Ortas, I. 2010. Effect of mycorrhiza application on plant growth and nutrient uptake in cucumber production under field conditions. Span. J. Agric. Res. 8(1), 116122. Doi: $10.5424 /$ sjar/201008S1-1230

Parra, S., G. Baca, J. Tirado, M. Villareal, P. Sánchez y S. Hernández. 2009. Calidad del fruto, composición y distribución de elementos minerales en pepino en respuesta a silicio y al potencial osmótico de la solución nutritiva. Terra Lat. 27 (2), 123-131.

Prajapati, K. y H. Modi. 2016. Growth promoting effect of potassium solubilizing Enterobacter hormaechei (KSB-8) on cucumber (Cucumis sativus) under hydroponic conditions. Int. J. Adv. Res. Biol. Sci. 3(5), 168-173.

Rauthan, B. y M. Schnitzer. 1981. Effects of a soil fulvic acid on the growth and nutrient content of cucumber (Cucumis sativus) plants. Plant Soil 63(3), 491-495. Doi: 10.1007/BF02370049

Reho, A. 2015. El pepino sinaloense continúa escalando su exportación. En: Meister Media Worldwide, http:// www.hortalizas.com/horticultura-protegida/el-pepino-sinaloense-continua-escalando-su-exportacion; consulta: agosto de 2016.

Sánchez, F., L. González, E. Moreno, J. Pineda y C. Reyes. 2014. Dinámica nutrimental y rendimiento de pepino cultivado en hidroponía con y sin recirculación de la solución nutritiva. Rev. Fitotec. Mex. 37(2), 261-269.

SAS Institute. 2008. Statistical analysis system. The SAS ${ }^{\circledR}$ system for Windows ${ }^{\circledR}$ version 9.1.3. Cary, NC, USA.

Sívori, M.E. 1986. Nutrición mineral. pp. 245-284. En: Sívori, E.M., E.R. Montaldi y O.H. Caso (eds.). Fisiología vegetal. Vol. II. Editorial Hemisferio Sur, Buenos Aires, Argentina.

Steiner, A. 1961. A universal method for preparing nutrient solutions of a certain desired composition. Plant Soil 15(2), 134-154. Doi: 10.1007/BF01347224

Valenzuela, H., R. Hamasaki y S. Fukuda. 1994. Field Cucumber production guidelines for Hawaii (No. 14488). HITAHR, College of Tropical Agriculture and Human Resources, University of Hawaii, Honolulu, HI, USA.

Van-Eerd, L. y K. O’Reilly. 2009. Yield, nitrogen dynamics, and fertilizer use efficiency in machine-harvested cucumber. HortScience 44(6), 1712-1718.

Zhang, Y. y H. Shi. 2008. Influences of phosphate deficiency in the medium on growth, activities of antioxidant enzymes and utilization of nitrogen resource in $\mathrm{Cu}$ cumis sativus hairy roots. Chin. J. Biotechnol. 24(9), 1604-1612.

Zhu, S., G. Wu, H. Cai, Z. Liu, J. Liu, R. Yang y X. Ai. 2015. Effects of low magnesium on photosynthesis characteristics and antioxidant system in cucumber seedlings under low temperature. Yingyong Shengtai Xuebao 26, 1351-1358. 